Research Article

\title{
Resonant Homoclinic Flips Bifurcation in Principal Eigendirections
}

\author{
Tiansi Zhang, ${ }^{1}$ Xiaoxin Huang, ${ }^{1}$ and Deming $\mathrm{Zhu}^{2}$ \\ ${ }^{1}$ College of Science, University of Shanghai for Science and Technology, Shanghai 200093, China \\ ${ }^{2}$ Department of Mathematics, East China Normal University, Shanghai 200062, China
}

Correspondence should be addressed to Tiansi Zhang; zhangts1209@163.com

Received 12 September 2013; Accepted 19 November 2013

Academic Editor: Svatoslav Staněk

Copyright (c) 2013 Tiansi Zhang et al. This is an open access article distributed under the Creative Commons Attribution License, which permits unrestricted use, distribution, and reproduction in any medium, provided the original work is properly cited.

A codimension-4 homoclinic bifurcation with one orbit flip and one inclination flip at principal eigenvalue direction resonance is considered. By introducing a local active coordinate system in some small neighborhood of homoclinic orbit, we get the Poincaré return map and the bifurcation equation. A detailed investigation produces the number and the existence of 1-homoclinic orbit, 1-periodic orbit, and double 1-periodic orbits. We also locate their bifurcation surfaces in certain regions.

\section{Introduction and Hypotheses}

The study of homoclinic flip bifurcations is comprehensively developed from the last two decades with the beginning work of Yanagida (1987) for homoclinic-doubling bifurcations. Generally there exist two kinds of homoclinic flips, namely the orbit flips and the inclination flips corresponding to nonprincipal homoclinic orbits or critically twisted homoclinic orbits, respectively. Kisaka et al. in $[1,2]$ and Naudot in [3] studied some cases of codimension two inclination flips; Morales and Pacifico in [4] and Naudot in [5] considered the orbit flips cases, while Homburg and Krauskopf in [6] proposed several unfoldings of the resonant homoclinic flip bifurcations around the central codimension-three point (the organizing centre) in parameter space to study the qualitative structure of bifurcation curves on a sphere and also that of Oldeman et al. in [7] by a numerical investigation with some software into these bifurcations in a specific threedimensional vector field.

Recently, Zhang et al. in [8-10] studied a kind of multiple flips homoclinic resonant bifurcation and got the existence of some saddle-node bifurcations and homoclinic-doubling bifurcations. Meanwhile Geng et al. in [11], Lu et al. in [12], and Liu in [13] discussed, respectively, a heterodimensional cycle flip or accompanied by transcritical bifurcation; they found the double and triple periodic orbit bifurcations and gave also some coexistence conditions for homoclinic orbits and periodic orbits.

As mentioned in [6,7], due to the break of three genericity conditions, there are many complicated homoclinic flips cases to study. In this paper, we confine our attention to a principal eigenvalue resonance of one orbit flip and one inclination flip homoclinic bifurcation. Compared with the above-mentioned work, our subject is very challenging and difficult because of the stronger degeneracy and the higher codimension. By constructing specifically a local active coordinate in a small tubular neighborhood of homoclinic orbit, we establish a regular map and then combine it with a singular map defined by the approximation solutions of system to build Poincaré return map (see also [14]). We obtain the existence of several 1-periodic orbit, 1-homoclinic orbit, and double 1-periodic orbits, as well as some bifurcation surfaces with the analysis of the bifurcation equation.

We first consider a $C^{r}$ system

$$
\dot{z}=f(z)+g(z, \mu)
$$

and its unperturbed system

$$
\dot{z}=f(z),
$$


where $r \geq 3, z \in \mathbb{R}^{4}, \mu \in \mathbb{R}^{l}, l \geq 4,0<|\mu| \ll 1, f(0)=0$, and $g(0, \mu)=g(z, 0)=0$. Suppose that the linearization $\mathrm{D} f(0)$ has four simple real eigenvalues $\lambda_{1}, \lambda_{2},-\rho_{1}$, and $-\rho_{2}$ with $\lambda_{2}>$ $\lambda_{1}>0>-\rho_{1}>-\rho_{2}$. Accordingly, the stable manifold $W^{s}$ and the unstable manifold $W^{u}$ are both two-dimensional. Let $W^{s s}$ and $W^{u u}$ be the strong stable manifold and the strong unstable manifold of the saddle $z=O$, respectively. Assume further that system (2) has a homoclinic orbit $\Gamma=\{z=r(t)$ : $t \in \mathbb{R}, r( \pm \infty)=0\}$. Hereinafter, our arguments will spread based on the following three hypotheses.

(H1) Resonance. $\lambda_{1}(\mu)=\rho_{1}(\mu),|\mu| \ll 1$, where $\lambda_{1}(0)=\lambda_{1}$ and $\rho_{1}(0)=\rho_{1}$.

(H2) Orbit Flip. Define $e^{+}=\lim _{t \rightarrow-\infty} \dot{r}(t) /|\dot{r}(t)| ; e_{s}^{-}=$ $\lim _{t \rightarrow+\infty} \dot{r}(t) /|\dot{r}(t)|$; then $e^{+} \in T_{0} W^{u}$ and $e_{s}^{-} \in T_{0} W^{s s}$ are unit eigenvectors corresponding to $\lambda_{1}$ and $-\rho_{2}$, respectively, where $T_{0} W^{u}$ (resp., $T_{0} W^{s s}$ ) is the tangent space of the corresponding manifold $W^{u}$ (resp., $W^{s s}$ ) at the saddle $z=O$.

(H3) Inclination Flip. Let $e_{u}^{+}$and $e^{-}$be the unit eigenvectors corresponding to $\lambda_{2}$ and $-\rho_{1}$, respectively, and

$$
\begin{aligned}
& \lim _{t \rightarrow+\infty}\left\{T_{r(t)} W^{u}, T_{r(t)} W^{s}, e_{u}^{+}\right\}=\mathbb{R}^{4}, \\
& \lim _{t \rightarrow-\infty}\left\{T_{r(t)} W^{u}, T_{r(t)} W^{s}, e^{-}\right\}=\mathbb{R}^{4} .
\end{aligned}
$$

Remark 1. Hypothesis (H2) is called an orbit flip because homoclinic orbit trends from the weak unstable manifold toward the strong stable manifold. Hypothesis (H3) means an inclination flip for its equivalence to

$$
\begin{aligned}
& T_{r(t)} W^{u} \longrightarrow \operatorname{span}\left\{e_{s}^{-}, e^{+}\right\}, \\
& T_{r(t)} W^{s} \longrightarrow \operatorname{span}\left\{e_{s}^{-}, e^{+}\right\} .
\end{aligned}
$$

\section{Poincaré Return Map}

This section treats mainly the establishment of Poincaré return map with two steps. To begin we first need to transform system (1) into a normal form in some neighborhood of the origin $O$.

It is well known that there are always two $C^{r}$ and $C^{r-1}$ transformations successively, also by the stable (or unstable) manifold theorem in [15], to straighten the local manifolds $W_{\mathrm{loc}}^{s}$ and $W_{\mathrm{loc}}^{u}$ as $W_{\mathrm{loc}}^{s}=\{z \in U, x=u=0\}$ and $W_{\mathrm{loc}}^{u}=\{z \epsilon$ $U, y=v=0\}$, respectively, $W_{\mathrm{loc}}^{s s}=\{z \in U, x=y=u=0\}$ (resp., $W_{\text {loc }}^{u u}=\{z \in U, x=y=v=0\}$ ); see [8-10]. Notice that now $\Gamma \cap W_{\text {loc }}^{u}=\{z \in U, u=u(x), y=v=0\}$ and $\Gamma \cap W_{\text {loc }}^{s}=$ $W_{\text {loc }}^{s s}$, where $z=(x, y, u, v) \in \mathbb{R}^{4}$ and $u(0)=u^{\prime}(0)=0$. Thus, system (1) can be changed to a $C^{r-2}$ form in the neighborhood $U$ as follows:

$$
\begin{aligned}
\dot{x}= & {\left[\lambda_{1}(\mu)+a(\mu) x v+o(|x v|)\right] x } \\
& +O(u)\left[O\left(x^{2} v\right)+O(y)\right], \\
\dot{y}= & {\left[-\rho_{1}(\mu)+b(\mu) x v+o(|x v|)\right] y } \\
& +O(v)[O(x y v)+O(u)],
\end{aligned}
$$

$$
\begin{aligned}
\dot{u}= & {\left[\lambda_{2}(\mu)+c(\mu) x v+o(|x v|)\right] u } \\
& +x^{2} H_{1}(x, y, v), \\
\dot{v}= & {\left[-\rho_{2}(\mu)+d(\mu) x v+o(|x v|)\right] v } \\
& +y^{2} H_{2}(x, y, u),
\end{aligned}
$$

where $H_{1}(x, 0,0)=0, H_{2}(0, y, 0)=0, \lambda_{1}(0)=\lambda_{1}, \lambda_{2}(0)=$ $\lambda_{2}, \rho_{1}(0)=\rho_{1}$, and $\rho_{2}(0)=\rho_{2} . a(\mu), b(\mu), c(\mu)$, and $d(\mu)$ are parameters depending on $\mu$.

Owing to the above straightness of the invariant manifolds, it is easy to find some moment $T$, such that $r(-T)=$ $\left\{\delta, 0, \delta_{u}, 0\right\}$ and $r(T)=\{0,0,0, \delta\}$ for some sufficiently small $\delta$ and $\left|\delta_{u}\right|=O\left(\delta^{2}\right)$ with $\{(x, y, u, v):|x|,|y|,|u|,|v|<2 \delta\} \subset U$. Wherefore one can choose

$$
\begin{gathered}
S_{0}=\{z=z(T):|x|,|y|,|u|,|v|<2 \delta\} \subset U, \\
S_{1}=\{z=z(-T):|x|,|y|,|u|,|v|<2 \delta\} \subset U,
\end{gathered}
$$

as the cross-sections of $\Gamma$ at $t=T$ and $t=-T$, respectively. Let $\tau$ be the time going from $q_{0}\left(x_{0}, y_{0}, u_{0}, v_{0}\right) \in S_{0}$ to $q_{1}\left(x_{1}, y_{1}, u_{1}, v_{1}\right) \in S_{1}$ and the Silnikov time $s=e^{-\lambda_{1}(\mu) \tau}$, with the help of the linear approximation solutions of system (5) (see [8-10]); we have thereby

$$
\begin{gathered}
x_{0}=x(T)=s x_{1}+O\left(x_{1}^{2} y_{0} s^{2} \ln s\right), \\
y_{1}=y(T+\tau)=s^{\rho_{1}(\mu) / \lambda_{1}(\mu)} y_{0}+O\left(x_{1} y_{0}^{2} s^{2} \ln s\right), \\
u_{0}=u(T) \\
=s^{\lambda_{2}(\mu) / \lambda_{1}(\mu)} u_{1}+O\left(x_{1} y_{0} u_{1} s^{\left(\lambda_{2}(\mu) / \lambda_{1}(\mu)\right)+1} \ln s\right), \\
v_{1}=v(T+\tau) \\
=s^{\rho_{2}(\mu) / \lambda_{1}(\mu)} v_{0}+O\left(x_{1} y_{0} v_{0} s^{\left(\rho_{2}(\mu) / \lambda_{1}(\mu)\right)+1} \ln s\right),
\end{gathered}
$$

which give explicitly the definition of the local transition map $F_{0}: S_{0} \rightarrow S_{1}: q_{0} \mapsto q_{1}$; see Figure 1(a).

In the following part we construct the map $F_{1}: S_{1} \rightarrow S_{0}$. Firstly consider the linear variational system

$$
\dot{z}=D f(r(t)) z
$$

and its adjoint system

$$
\dot{z}=-(D f(r(t)))^{*} z .
$$




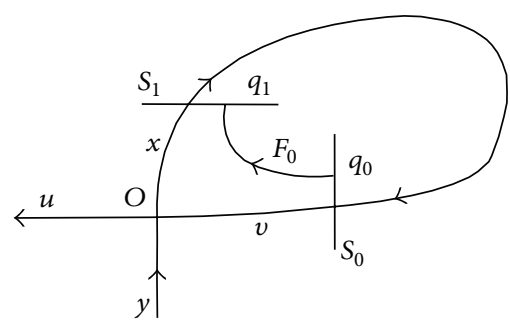

(a) $F_{0}: S_{0} \rightarrow S_{1}$



(b) $F_{1}: S_{1} \rightarrow S_{0}$

Figure 1: Transition maps.

Lemma 2. There exists a fundamental solution matrix $Z(t)=$ $\left(z_{1}(t), z_{2}(t), z_{3}(t), z_{4}(t)\right)$ of system (8) satisfying

$$
\begin{gathered}
Z(-T)=\left(\begin{array}{cccc}
w_{11} & w_{21} & 0 & w_{41} \\
w_{12} & 0 & 0 & w_{42} \\
w_{13} & w_{23} & 1 & w_{43} \\
0 & 0 & 0 & w_{44}
\end{array}\right), \\
Z(T)=\left(\begin{array}{cccc}
0 & 0 & w_{31} & 0 \\
w_{14} & 0 & w_{32} & 1 \\
1 & 0 & w_{33} & 0 \\
0 & 1 & w_{34} & 0
\end{array}\right),
\end{gathered}
$$

where $z_{1}(t) \in\left(T_{r(t)} W^{u}\right)^{c} \cap\left(T_{r(t)} W^{s}\right)^{c}, z_{2}(t)=-\dot{r}(t) /\left|\dot{r}^{y}(T)\right| \epsilon$ $T_{r(t)} W^{u} \cap T_{r(t)} W^{s}, z_{3}(t) \in T_{r(t)} W^{u}, z_{4}(t) \in T_{r(t)} W^{s}$ and $w_{12} w_{21} w_{31} w_{44} \neq 0, w_{23}<0$. Moreover, $\left|w_{1 i} w_{12}^{-1}\right| \ll 1, i \neq 2$, $\left|w_{3 i} w_{31}^{-1}\right| \ll 1, i \neq 1,\left|w_{4 i} w_{44}^{-1}\right| \ll 1, i \neq 4$ for $T \rightarrow+\infty$.

Proof. Notice that the tangent subspace $T_{\gamma(-T)} W^{u}$ is invariant and $W_{\text {loc }}^{u u} \cap U$ is straightened to be $u$ axis; it is possible to choose $z_{3}(-T)=(0,0,1,0)$ since $z_{3}(t) \in T_{\gamma(t)} W^{u}$. While for $w_{31} \neq 0$, it is because $\lim _{t \rightarrow+\infty} T_{\gamma(t)} W^{u}=\operatorname{span}\left\{e^{+}, e_{s}^{-}\right\}$and $z_{3}(T) \in T_{\gamma(T)} W^{u}$ corresponding to $x$ axis.

As to $z_{i}(-T)$ or $z_{i}(T), i=1,2,4$, one may refer to $[8,9]$ for the similar proof, but we omit the details here.

Remark 3. The matrix $\left(Z^{-1}(t)\right)^{*}$ is a fundamental solution matrix of system (9). We denote it as $\Phi(t)=$ $\left(\phi_{1}(t), \phi_{2}(t), \phi_{3}(t), \phi_{4}(t)\right)=\left(Z^{-1}(t)\right)^{*}$; then $\phi_{1}(t) \quad \epsilon$ $\left(T_{r(t)} W^{u}\right)^{c} \cap\left(T_{r(t)} W^{s}\right)^{c}$ is bounded and tends to zero exponentially as $|t| \rightarrow+\infty$ due to $<\phi_{i}(t), z_{i}(t) \geq 1$ and $z_{i}(t)$ tends exponentially to infinity.

In fact $\left(z_{1}(t), z_{2}(t), z_{3}(t), z_{4}(t)\right)$ can be regarded as a new local coordinate system along $\Gamma$. So we may make a coordinate change as

$$
\begin{aligned}
s(t) & =r(t)+Z(t) N \\
& =r(t)+z_{1}(t) n_{1}+z_{3}(t) n_{3}+z_{4}(t) n_{4},
\end{aligned}
$$

where $N=N(t)=\left(n_{1}(t), 0, n_{3}(t), n_{4}(t)\right)^{*}$. Note that the new $s(t)$ should satisfy system (1); that is,

$$
\begin{aligned}
\dot{s}(t) & =f(s(t))+g(s(t), \mu) \\
& =f(r(t)+Z(t) N)+g(r(t)+Z(t) N, \mu) .
\end{aligned}
$$

An asymptotic expansion with respect to $r(t)$ shows that

$$
\begin{aligned}
\dot{n}_{i}= & \phi_{i}^{*}(t) g_{\mu}(r(t), 0) \mu+O\left(|\mu|^{2}\right) \\
& +O\left(|N|^{2}\right)+O(|\mu||N|), \quad i=1,3,4 .
\end{aligned}
$$

Via integrating both sides from $-T$ to $T$ of this equation, one can finally obtain

$$
n_{i}(T)=n_{i}(-T)+M_{i} \mu+\text { h.o.t. }, \quad i=1,3,4,
$$

where $M_{i}=\int_{-T}^{T} \phi_{i}^{*}(t) g_{\mu}(r(t), 0) \mathrm{d} t, i=1,3,4$ are the Melnikov vectors. And further $M_{1}=\int_{-T}^{T} \phi_{1}^{*}(t) g_{\mu}(r(t), 0) \mathrm{d} t=$ $\int_{-\infty}^{+\infty} \phi_{1}^{*}(t) g_{\mu}(r(t), 0) \mathrm{d} t$.

Equation (14) defines exactly the map $F_{1}: S_{1} \rightarrow S_{0}$; $N(-T) \mapsto N(T)$ under the new coordinate system; see Figure 1(b).

In order to combine $F_{0}$ and $F_{1}$ into the Poincaré return map, we still need to establish a relationship between the original and the new coordinate systems. In doing so, recall that $z(t)=r(t)+Z(t) N(t)$; then by taking time $t=T$ and $t=-T$, respectively, together with $z(T)=q_{2 j}\left(x_{2 j}, y_{2 j}, u_{2 j}\right.$, $\left.v_{2 j}\right) \in S_{0}, z(-T)=q_{2 j+1}\left(x_{2 j+1}, y_{2 j+1}, u_{2 j+1}, v_{2 j+1}\right) \in S_{1}$ and $N_{2 j}(T)=\left(n_{2 j, 1}, 0, n_{2 j, 3}, n_{2 j, 4}\right), N_{2 j+1}(-T)=\left(n_{2 j+1,1}, 0, n_{2 j+1,3}\right.$, $\left.n_{2 j+1,4}\right), j=0,1,2, \ldots$, we obtain immediately the following formulas:

$$
\begin{gathered}
n_{2 j, 1}=u_{2 j}-w_{33} w_{31}^{-1} x_{2 j}, \\
n_{2 j, 3}=w_{31}^{-1} x_{2 j}, \\
n_{2 j, 4}=y_{2 j}-w_{14} u_{2 j}+\left(w_{14} w_{33}-w_{32}\right) w_{31}^{-1} x_{2 j}, \\
n_{2 j+1,1}=w_{12}^{-1} y_{2 j+1}-w_{42} w_{12}^{-1} w_{44}^{-1} v_{2 j+1}, \\
n_{2 j+1,3}=u_{2 j+1}-\delta_{u}-w_{13} w_{12}^{-1} y_{2 j+1} \\
+\left(w_{13} w_{42} w_{12}^{-1}-w_{43}\right) w_{44}^{-1} v_{2 j+1}, \\
n_{2 j+1,4}=w_{44}^{-1} v_{2 j+1}, \\
x_{2 j+1} \approx \delta, \quad v_{2 j} \approx \delta .
\end{gathered}
$$


With all of the above, the Poincare return map is given as $F=F_{1} \circ F_{0}$. Therefore, the associated successor function $G(s$, $\left.u_{1}, y_{0}\right)=\left(G_{1}, G_{3}, G_{4}\right)=F\left(q_{0}\right)-q_{0}$ is

$$
\begin{aligned}
G_{1}= & w_{12}^{-1} s^{\rho_{1}(\mu) / \lambda_{1}(\mu)} y_{0}-u_{1} s^{\lambda_{2}(\mu) / \lambda_{1}(\mu)}+w_{33} w_{31}^{-1} \delta s \\
& -w_{42} w_{12}^{-1} w_{44}^{-1} \delta s^{\rho_{2}(\mu) / \lambda_{1}(\mu)}+M_{1} \mu+\text { h.o.t., } \\
G_{3}= & u_{1}-\delta_{u}-w_{13} w_{12}^{-1} s y_{0}-w_{31}^{-1} \delta s \\
& +\left(w_{13} w_{42} w_{12}^{-1}-w_{43}\right) w_{44}^{-1} \delta s^{\rho_{2}(\mu) / \lambda_{1}(\mu)} \\
& +M_{3} \mu+\text { h.o.t., } \\
G_{4}= & w_{44}^{-1} \delta s^{\rho_{2}(\mu) / \lambda_{1}(\mu)}-y_{0}+w_{14} s^{\lambda_{2}(\mu) / \lambda_{1}(\mu)} u_{1} \\
& +\left(w_{32}-w_{14} w_{33}\right) w_{31}^{-1} \delta s+M_{4} \mu+\text { h.o.t.. }
\end{aligned}
$$

\section{Bifurcation Results}

From the definition of the Silnikov time $s=e^{-\lambda_{1}(\mu) \tau}$, we know that a solution with $s>0$ of (16) or equivalently $\tau>0$ corresponds to a periodic orbit near $\Gamma$ and a solution with $s=0$ of (16) or equivalently $\tau=+\infty$ corresponds to a homoclinic orbit near $\Gamma$. It is enough to study the solutions of the successor function $G\left(s, u_{1}, y_{0}\right)=0$ for bifurcation analysis. Consider for concision that we omit the dependence on parameter $\mu$ from now on in the exponent notation.

From $G_{3}=0$ and $G_{4}=0$, there are

$$
\begin{aligned}
u_{1}= & \delta_{u}+w_{31}^{-1} \delta s-M_{3} \mu \\
& +O\left(s y_{0}\right)+O\left(s^{\rho_{2} / \lambda_{1}}\right), \\
y_{0}= & \left(w_{32}-w_{33} w_{14}\right) w_{31}^{-1} \delta s \\
& +M_{4} \mu+O\left(s^{\rho_{2} / \lambda_{1}}\right)+O\left(s^{\lambda_{2} / \lambda_{1}}\right) .
\end{aligned}
$$

Put $u_{1}$ and $y_{0}$ into $G_{1}=0$; we have

$$
\begin{aligned}
F(s, \mu) \equiv & M_{4} \mu s-w_{42} w_{44}^{-1} \delta s^{\rho_{2} / \lambda_{1}}+w_{12} w_{33} w_{31}^{-1} \delta s \\
& -\delta_{u} w_{12} s^{\lambda_{2} / \lambda_{1}}+w_{12} M_{3} \mu s^{\lambda_{2} / \lambda_{1}} \\
& +\left(w_{32}-w_{33} w_{14}\right) w_{31}^{-1} \delta s^{2}+w_{44}^{-1} \delta s^{\left(\rho_{1}+\rho_{2}\right) / \lambda_{1}} \\
& -w_{12} w_{31}^{-1} \delta s^{1+\left(\lambda_{2} / \lambda_{1}\right)}+w_{12} M_{1} \mu+w_{14} \delta_{u} s^{\left(\rho_{1}+\lambda_{2}\right) / \lambda_{1}} \\
& +w_{13} M_{4} \mu s^{\left(\lambda_{1}+\lambda_{2}\right) / \lambda_{1}}+\text { h.o.t. }=0,
\end{aligned}
$$

which is the bifurcation equation. Furthermore, for $w_{33} \neq 0$,

$$
\begin{aligned}
& \left.\operatorname{det} \widetilde{G}\right|_{\substack{Q=0 \\
\mu=0}} \\
& \quad=\left|\begin{array}{ccc}
w_{33} w_{31}^{-1} \delta & 0 & 0 \\
-\delta w_{31}^{-1} & 1 & 0 \\
\left(w_{32}-w_{33} w_{14}\right) w_{31}^{-1} \delta & 0 & -1
\end{array}\right| \neq 0,
\end{aligned}
$$

where $\widetilde{G}=\partial\left(G_{1}, G_{3}, G_{4}\right) / \partial Q\left(s, u_{1}, y_{0}\right)$. Implicit function theorem reveals that (16) has a unique solution as

$$
s=s(\mu), \quad u_{1}=u_{1}(\mu), \quad y_{0}=y_{0}(\mu),
$$

with $s(0)=0, u_{1}(0)=0$, and $y_{0}(0)=0$. It means that system (1) has a unique periodic orbit as $s>0$ or a unique homoclinic orbit as $s=0$, and they cannot coexist.

Theorem 4. Suppose that $M_{1} \neq 0$ and $w_{33} \neq 0$ are true; then system (1) has a unique 1-periodic orbit near $\Gamma$ for $w_{31} w_{33} M_{1} \mu<0$ or has a unique 1-homoclinic orbit $\Gamma_{\mu}$ near $\Gamma$ as $\mu \in H^{1} \stackrel{\Delta}{=}\left\{\mu: M_{1} \mu+\right.$ h.o.t. $\left.=0\right\}$, and they do not coexist.

Proof. Clearly $F(s, \mu)=0$ has a small positive solution $s=$ $-\delta^{-1} w_{33}^{-1} w_{31} M_{1} \mu+$ h.o.t., for $w_{31} w_{33} M_{1} \mu<0$, and has a zero solution $s=0$ for $\mu \in H^{1}$ which is a codimension-one hypersurface.

In the following part we restrict our attention on the case $w_{33}=0$ for $2 \lambda_{1}>\lambda_{2}>\rho_{2}$. Define

$$
\begin{gathered}
R_{i j}^{k l}=\left\{\mu:(-1)^{k}\left(w_{12}\right)^{l} M_{i} \mu M_{j} \mu>0\right\}, \\
E_{j}^{k l}=\left\{\mu:(-1)^{k}\left(w_{12}\right)^{l} w_{42} w_{44} M_{j} \mu>0\right\},
\end{gathered}
$$

where $k, l=0,1, i=1,3, j=1,3,4$.

In order to well solve (18), we rewrite it into two parts, namely, a line $W=P(s, \mu)$ and a curve $W=Q(s, \mu)$ with respect to $s$ :

$$
\begin{aligned}
& P(s, \mu)=M_{4} \mu s+w_{12} M_{1} \mu+\text { h.o.t., } \\
& Q(s, \mu)=w_{42} w_{44}^{-1} \delta s^{\rho_{2} / \lambda_{1}}+\delta_{u} w_{12} s^{\lambda_{2} / \lambda_{1}}-w_{12} M_{3} \mu s^{\lambda_{2} / \lambda_{1}} \\
& -w_{32} w_{31}^{-1} \delta s^{2}-w_{44}^{-1} \delta s^{\left(\rho_{1}+\rho_{2}\right) / \lambda_{1}} \\
& +w_{12} w_{31}^{-1} \delta s^{\left(\lambda_{1}+\lambda_{2}\right) / \lambda_{1}}+\text { h.o.t.. }
\end{aligned}
$$

Then there are firstly the following conclusions based on an analysis of the relative position of the line $W=P(s, \mu)$ and the curve $W=Q(s, \mu)$.

Lemma 5. Suppose that $2 \lambda_{1}>\lambda_{2}>\rho_{2}, w_{33}=0$, and $w_{42} \neq 0$ hold; then $F(s, \mu)=0$ has a unique small positive solution $\widetilde{s} \in\left(0, s^{*}\right)$ for $\mu \in E_{1}^{01}$, where $s^{*}=\left[w_{44} w_{42}^{-1} \delta\left(2 w_{12} M_{1} \mu+\right.\right.$ $\left.\left.M_{4} \mu\right)\right]^{\lambda_{1} / \rho_{2}}$.

Proof. It is clear that

$$
\begin{gathered}
P(0, \mu)=w_{12} M_{1} \mu+\text { h.o.t., } \quad Q(0, \mu)=0, \\
P^{\prime}(s, \mu)=M_{4} \mu+\text { h.o.t., } \\
Q^{\prime}(s, \mu)=\rho_{2} \lambda_{1}^{-1} w_{42} w_{44}^{-1} \delta s^{\left(\rho_{2} / \lambda_{1}\right)-1}+\text { h.o.t.. }
\end{gathered}
$$

Therefore, the line $W=P(s, \mu)$ intersects the curve $W=$ $Q(s, \mu)$ at a unique point $\widetilde{s}$ for $\mu \in E_{1}^{01}$. Notice that $Q\left(s^{*}, \mu\right)=$ $2 w_{12} M_{1} \mu+M_{4} \mu+$ h.o.t. $>w_{12} M_{1} \mu+M_{4} \mu s^{*}+$ h.o.t. $=P\left(s^{*}, \mu\right)$, so $\widetilde{s} \in\left(0, s^{*}\right)$. 
<smiles>[Y19]C1OC1[As]</smiles>

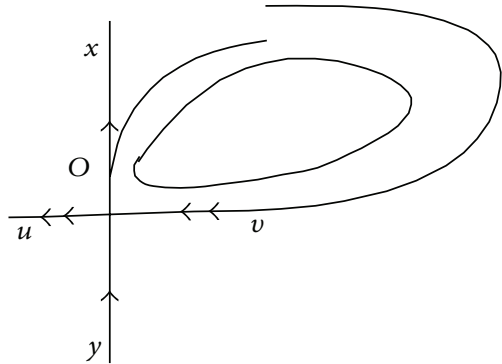

(a) Double 1-periodic orbit
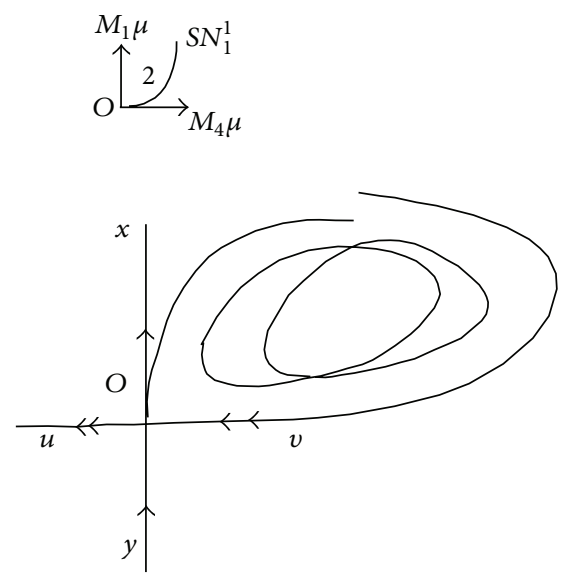

(b) Two 1-periodic orbits
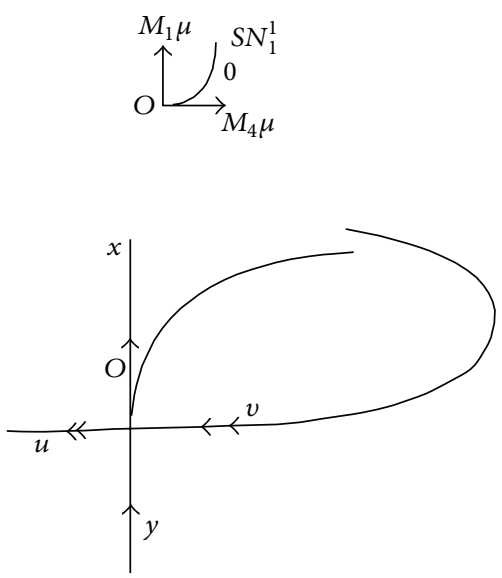

(c) No 1-periodic orbit

Figure 2: $\operatorname{Rank}\left(M_{1}, M_{4}\right)=2$ and $\mu \in E_{1}^{11} \cap R_{14}^{11}$.

Theorem 6. Suppose that $2 \lambda_{1}>\lambda_{2}>\rho_{2}, w_{33}=0$, and $w_{42} \neq 0$ hold; then system (1) has exactly a unique (resp., not any) 1periodic orbit for $\mu \in E_{1}^{01}$ (resp., $\mu \in E_{1}^{11} \cap R_{14}^{01}$ ).

Proof. From Lemma 5, we know that $F(s, \mu)=0$ has exactly a unique small positive solution for $\mu \in E_{1}^{01}$ which corresponds exactly to a 1-periodic orbit of system (1). Moreover, $F(s, \mu)=$ 0 does not have any small positive solutions for $\mu \in E_{1}^{11} \cap$ $R_{14}^{01}$.

Theorem 7. Suppose that $2 \lambda_{1}>\lambda_{2}>\rho_{2}, w_{33}=0$, and $w_{42} \neq 0$ hold; then, for $\mu \in E_{1}^{11} \cap R_{14}^{11}$ and Rank $\left(M_{1}, M_{4}\right)=2$, system (1) has a unique double 1-periodic orbit near $\Gamma$ on the bifurcation surface

$$
\begin{gathered}
S N^{1}: w_{12} M_{1} \mu+\frac{\rho_{2}-\lambda_{1}}{\rho_{2}}\left(\frac{\lambda_{1} w_{44}}{\rho_{2} w_{42} \delta}\right)^{\lambda_{1} /\left(\rho_{2}-\lambda_{1}\right)} \\
\times\left(M_{4} \mu\right)^{\rho_{2} /\left(\rho_{2}-\lambda_{1}\right)}+\text { h.o.t. }=0,
\end{gathered}
$$

which has a normal vector $M_{1}$ at $\mu=0$. The corresponding double positive zero point is

$$
s_{*}=\left(\frac{\lambda_{1} w_{44} M_{4} \mu}{\rho_{2} w_{42} \delta}\right)^{\lambda_{1} /\left(\rho_{2}-\lambda_{1}\right)}+\text { h.o.t. }
$$

as $\mu \in E_{4}^{00}$ (see Figure 2(a)).

Proof. We know that the existence of a double 1-periodic orbit corresponds to the equations $P(s, \mu)=Q(s, \mu), P^{\prime}(s, \mu)=$ $Q^{\prime}(s, \mu)$, and $P^{\prime \prime}(s, \mu) \neq Q^{\prime \prime}(s, \mu)$, that is,

$$
\begin{aligned}
M_{4} \mu s+w_{12} M_{1} \mu= & w_{42} w_{44}^{-1} \delta s^{\rho_{2} / \lambda_{1}}+\delta_{u} w_{12} s^{\lambda_{2} / \lambda_{1}} \\
& -w_{12} M_{3} \mu s^{\lambda_{2} / \lambda_{1}}-w_{32} w_{31}^{-1} \delta s^{2} \\
& -w_{44}^{-1} \delta s^{\left(\rho_{1}+\rho_{2}\right) / \lambda_{1}}+w_{12} w_{31}^{-1} \delta s^{\left(\lambda_{1}+\lambda_{2}\right) / \lambda_{1}} \\
& + \text { h.o.t. }
\end{aligned}
$$

$$
\begin{aligned}
M_{4} \mu= & \frac{\rho_{2}}{\lambda_{1}} w_{42} w_{44}^{-1} \delta s^{\left(\rho_{2} / \lambda_{1}\right)-1}+\frac{\lambda_{2}}{\lambda_{1}} \delta_{u} w_{12} s^{\left(\lambda_{2} / \lambda_{1}\right)-1} \\
& -\frac{\lambda_{2}}{\lambda_{1}} w_{12} M_{3} \mu s^{\left(\lambda_{2} / \lambda_{1}\right)-1}-2 w_{32} w_{31}^{-1} \delta s \\
& -\frac{\rho_{1}+\rho_{2}}{\lambda_{1}} w_{44}^{-1} \delta s^{\rho_{2} / \lambda_{1}} \\
& +\frac{\lambda_{1}+\lambda_{2}}{\lambda_{1}} w_{12} w_{31}^{-1} \delta s^{\lambda_{2} / \lambda_{1}}+\text { h.o.t. } \\
0 \neq & \rho_{2}\left(\rho_{2}-\lambda_{1}\right) w_{42} w_{44}^{-1} \delta s^{\left(\rho_{2} / \lambda_{1}\right)-2} \\
& +\lambda_{2}\left(\lambda_{2}-\lambda_{1}\right) \delta_{u} w_{12} s^{\left(\lambda_{2} / \lambda_{1}\right)-2} \\
& -\lambda_{2}\left(\lambda_{2}-\lambda_{1}\right) w_{12} M_{3} \mu s^{\left(\lambda_{2} / \lambda_{1}\right)-2} \\
& -2 \lambda_{1}^{2} w_{32} w_{31}^{-1} \delta \\
& +\rho_{2}\left(\rho_{1}+\rho_{2}\right) w_{44}^{-1} \delta s^{\left(\rho_{2} / \lambda_{1}\right)-1} \\
& +\lambda_{2}\left(\lambda_{1}+\lambda_{2}\right) w_{12} w_{31}^{-1} \delta s^{\left(\lambda_{2} / \lambda_{1}\right)-1}+\text { h.o.t. }
\end{aligned}
$$

having solutions. Indeed, the second equation of (26) permits the double positive zero point $s_{*}$ as $\mu \in E_{4}^{00}$. Putting it into the first equation of (26), there is

$$
\begin{aligned}
& M_{4} \mu\left(\frac{\lambda_{1} w_{44} M_{4} \mu}{\rho_{2} w_{42} \delta}\right)^{\lambda_{1} /\left(\rho_{2}-\lambda_{1}\right)}+w_{12} M_{1} \mu \\
& \quad=w_{42} w_{44}^{-1} \delta\left(\frac{\lambda_{1} w_{44} M_{4} \mu}{\rho_{2} w_{42} \delta}\right)^{\rho_{2} /\left(\rho_{2}-\lambda_{1}\right)}+\text { h.o.t.. }
\end{aligned}
$$

Then $S N^{1}$ exists for $\mu \in E_{1}^{11} \cap R_{14}^{11}$.

From the above proof, we see that, when $M_{4} \mu>0$ and $w_{12} M_{1} \mu<0$, the line $W=P(s, \mu)$ has a positive slope lying under the curve $W=Q(s, \mu)$ when $w_{42} w_{44}>0$, so if $w_{12} M_{1} \mu$ 


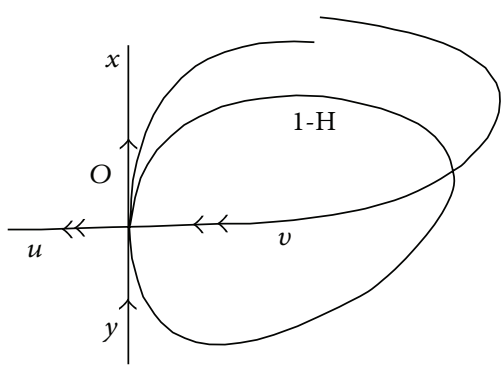

(a) $F(0, \mu)=0, y_{0}=M_{4} \mu+$ h.o.t. $\neq 0$

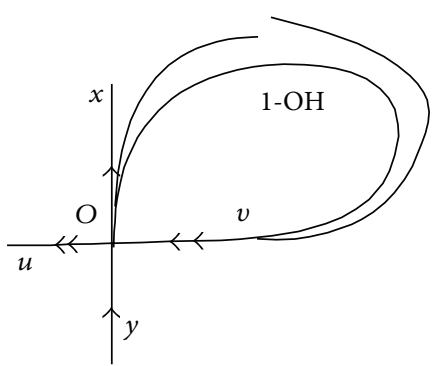

(b) $F(0, \mu)=0, y_{0}=M_{4} \mu+$ h.o.t. $=0$

Figure 3: 1-homoclinic orbit (1-H) and (1-OH).

increases, the line must intersect the curve at two sufficiently small positive points, which can be equal to the existence of two 1-periodic orbits of system (1). For $M_{4} \mu<0, w_{42} w_{44}<$ 0 , and $w_{12} M_{1} \mu>0$, the arguments are similar. So we have immediately a complement of Theorem 7 .

Corollary 8. Assume that the hypotheses of Theorem 7 are valid, system (1) then has two (resp., not any) 1-periodic orbits near $\Gamma$ when $\mu$ lies on the side of $S N^{1}$ which points to the direction ( $\left.\operatorname{sgn} w_{12} w_{42} w_{44}\right) M_{1}$ (resp., in the opposite direction) (see Figures 2(b) and 2(c)).

As Melnikov functions generally play an important role in bifurcation study, the following theorem shows also the existence of some double 1-periodic orbits relying on the investigation of $M_{i}=0$ for $i=1,3,4$.

Theorem 9. Suppose $2 \lambda_{1}>\lambda_{2}>\rho_{2}, w_{33}=0$, and $w_{42} \neq 0$ are valid; then the following applies.

(1) For $M_{1}=0$ or $M_{1}^{2}+M_{3}^{2}=0$, system (1) has exactly one 1-homoclinic orbit and one (resp., not any) 1-periodic orbit near $\Gamma$ and they (resp., do not) coexist as $\mu \in E_{4}^{00}$ (resp., $\mu \in E_{4}^{10}$ ).

(2) For $M_{3}=0$, system (1) has exactly one (resp., not any) 1-periodic orbit near $\Gamma$ as $\mu \in E_{1}^{01}$ (resp., $\mu \in E_{1}^{11} \cap R_{14}^{01}$ ). system (1) has a unique double 1-periodic orbit near $\Gamma$ as $\mu \in E_{1}^{11} \cap R_{14}^{11}$ and $\operatorname{Rank}\left(M_{1}, M_{4}\right)=2$. Accordingly, the double 1-periodic orbit bifurcation surface is $S N^{1}: w_{12} M_{1} \mu+\left(\left(\rho_{2}-\right.\right.$ $\left.\left.\lambda_{1}\right) w_{42} \delta / \lambda_{1} w_{44}\right)\left(\lambda_{1} w_{44} M_{4} \mu / \rho_{2} \delta w_{42}\right)^{\rho_{2} /\left(\rho_{2}-\lambda_{1}\right)}+$ h.o.t. $=0$ with a normal vector $M_{1}$ at $\mu=0$, and it may generate two 1-periodic orbits when $\mu$ lies in the direction $\left(\operatorname{sgn} w_{42} w_{44} w_{12}\right) M_{1}$ of $S N^{1}$ and no such a 1-periodic orbit in the opposite direction.

(3) For $M_{4}=0$ or $M_{3}^{2}+M_{4}^{2}=0$, system (1) has only one (resp. not any) 1-periodic orbit near $\Gamma$ as $\mu \in E_{1}^{01}$ (resp., $\left.\mu \in E_{1}^{11}\right)$.

(4) For $M_{1}^{2}+M_{4}^{2}=0$, system (1) does not have any 1periodic orbit near $\Gamma$.

(5) For $M_{1}^{2}+M_{3}^{2}+M_{4}^{2}=0$, system (1) has only one 1homoclinic orbit near $\Gamma$.
Proof. When $M_{1}=0$ or $M_{1}=M_{3}=0, F(s, \mu)=s\left(M_{4} \mu-\right.$ $w_{42} w_{44}^{-1} \delta s^{\left(\rho_{2}-\lambda_{1}\right) / \lambda_{1}}-\delta_{u} w_{12} s^{\left(\lambda_{2}-\lambda_{1}\right) / \lambda_{1}}+w_{12} M_{3} \mu s^{\left(\lambda_{2}-\lambda_{1}\right) / \lambda_{1}}+$ $w_{32} w_{31}^{-1} \delta s+w_{44}^{-1} \delta s^{\rho_{2} / \lambda_{1}}+$ h.o.t. $)=0$ has two solutions $s_{1}=0$ and $s_{2}=\left(w_{44} M_{4} \mu / w_{42} \delta\right)^{\lambda_{1} /\left(\rho_{2}-\lambda_{1}\right)}+$ h.o.t. for $\mu \in E_{4}^{00}$ which correspond to a 1-periodic orbit, and a 1-homoclinic orbit respectively. Thus, (1) is true.

The result of the cases (2) is exactly the same as that of Theorem 7.

If $M_{4}=0$ or $M_{3}=M_{4}=0, F(s, \mu)=0$ has only a positive solution $s_{3}=\left(w_{44} w_{12} M_{1} \mu / \delta w_{42}\right)^{\lambda_{1} / \rho_{2}}+$ h.o.t. for $\mu \in E_{1}^{01}$, which means system (1) has a 1-periodic orbit. Then (3) is valid.

In case of $M_{1}^{2}+M_{4}^{2}=0$, it is clear that $F(s, \mu)=0$ does not have any small nonnegative solutions, so system (1) does not have, any 1-homoclinic orbits or 1-periodic orbits.

The last conclusion is obvious. Thereby, the proof is complete.

Remark 10. Notice that, in Theorem 9 (1) and (5), $F(s, \mu)=0$ has a solution $s=0$, which means that system (1) has a codimension-1 1-homoclinic orbit (see Figure 3(a)), so the existing homoclinic orbit is no longer orbit flip for $y_{0}=$ $M_{4} \mu+$ h.o.t. $\neq 0$. But if $y_{0}=0$, an orbit flip homoclinic orbit could still exist, where $y_{0}$ is given by $G_{4}=0$; see Figure 3(b).

Remark 11. There still exist some double 1-periodic orbits or triple 1-periodic orbits for the case $w_{42}=0$ and $\delta_{u} \neq 0$; one may pursue the similar process to discuss, so we leave it here.

\section{Acknowledgment}

The project is supported by the National Natural Science Foundation of China (no. 11126097).

\section{References}

[1] M. Kisaka, H. Kokubu, and H. Oka, "Bifurcations to N-homoclinic orbits and $\mathrm{N}$-periodic orbits in vector fields," Journal of Dynamics and Differential Equations, vol. 5, no. 2, pp. 305-357, 1993.

[2] M. Kisaka, H. Kokubu, and H. Oka, "Supplement to homoclinic-doubling bifurcation in vector fields," in Handbook of Dynamical Systems, pp. 92-116, 1993. 
[3] V. Naudot, "Strange attractor in the unfolding of an inclinationflip homoclinic orbit," Ergodic Theory and Dynamical Systems, vol. 16, no. 5, pp. 1071-1086, 1996.

[4] C. A. Morales and M. J. Pacifico, "Inclination-flip homoclinic orbits arising from orbit-flip," Nonlinearity, vol. 14, no. 2, pp. 379-393, 2001.

[5] V. Naudot, "A strange attractor in the unfolding of an orbit-flip homoclinic orbit," Dynamical Systems, vol. 17, no. 1, pp. 45-63, 2002.

[6] A. J. Homburg and B. Krauskopf, "Resonant homoclinic flip bifurcations," Journal of Dynamics and Differential Equations, vol. 12, no. 4, pp. 807-850, 2000.

[7] B. E. Oldeman, B. Krauskopf, and A. R. Champneys, "Numerical unfoldings of codimension-three resonant homoclinic flip bifurcations," Nonlinearity, vol. 14, no. 3, pp. 597-621, 2001.

[8] T. S. Zhang and D. M. Zhu, "Homoclinic bifurcation of orbit flip with resonant principal eigenvalues," Acta Mathematica Sinica, vol. 22, no. 3, pp. 855-864, 2006.

[9] T. Zhang and D. Zhu, "Bifurcations of homoclinic orbit connecting two nonleading eigendirections," International Journal of Bifurcation and Chaos in Applied Sciences and Engineering, vol. 17, no. 3, pp. 823-836, 2007.

[10] T. S. Zhang and D. M. Zhu, "Bifurcation analysis of homoclinic flips at principal eigenvalues resonance," Applied Mathematics, vol. 4, pp. 271-278, 2013.

[11] F. Geng, D. Liu, and D. Zhu, "Bifurcations of generic heteroclinic loop accompanied by transcritical bifurcation," International Journal of Bifurcation and Chaos in Applied Sciences and Engineering, vol. 18, no. 4, pp. 1069-1083, 2008.

[12] Q. Lu, Z. Qiao, T. Zhang, and D. Zhu, "Heterodimensional cycle bifurcation with orbit-flip," International Journal of Bifurcation and Chaos in Applied Sciences and Engineering, vol. 20, no. 2, pp. 491-508, 2010.

[13] X. Liu, "Homoclinic flip bifurcations accompanied by transcritical bifurcation," Chinese Annals of Mathematics B, vol. 32, no. 6, pp. 905-916, 2011.

[14] D. Zhu and Z. Xia, "Bifurcations of heteroclinic loops," Science in China A, vol. 41, no. 8, pp. 837-848, 1998.

[15] S. Wiggins, Global Bifurcations and Chaos, vol. 73, Springer, New York, NY, USA, 1988. 


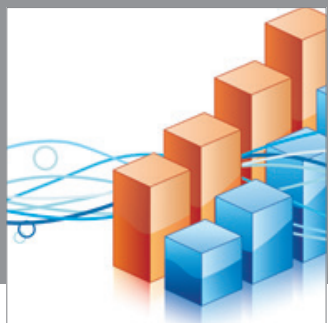

Advances in

Operations Research

mansans

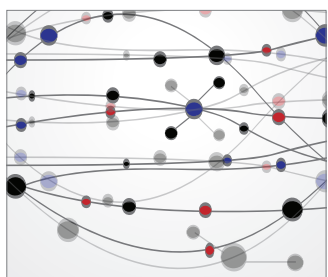

The Scientific World Journal
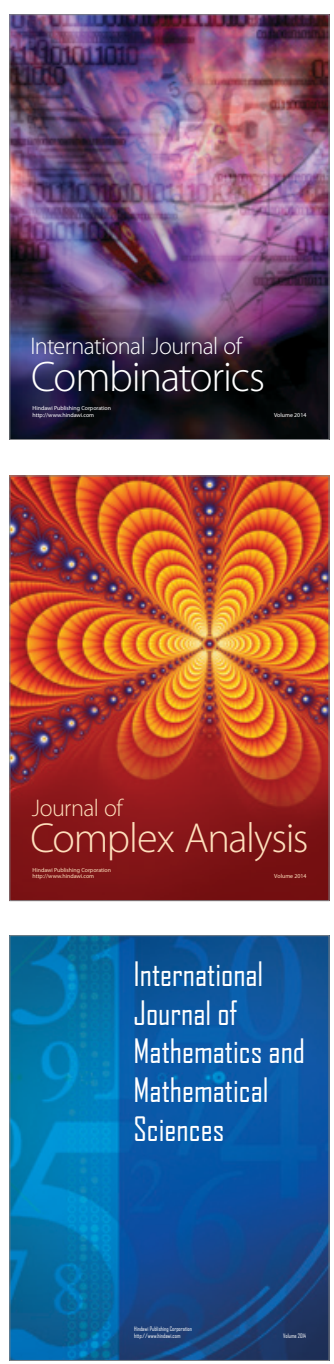


Submit your manuscripts at http://www.hindawi.com
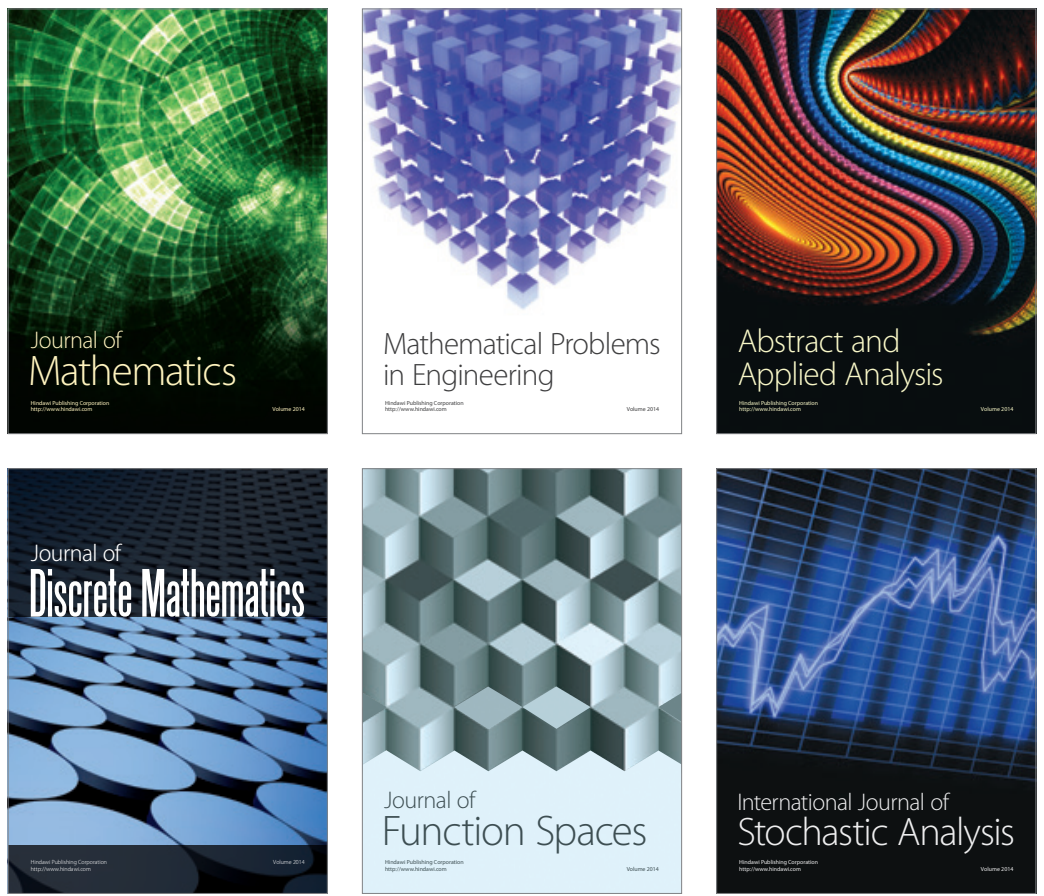

Journal of

Function Spaces



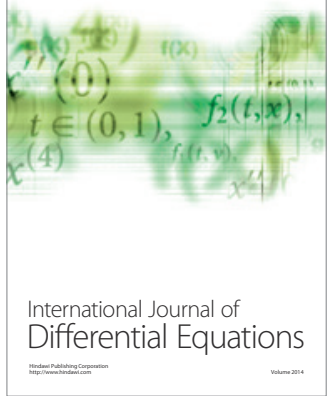
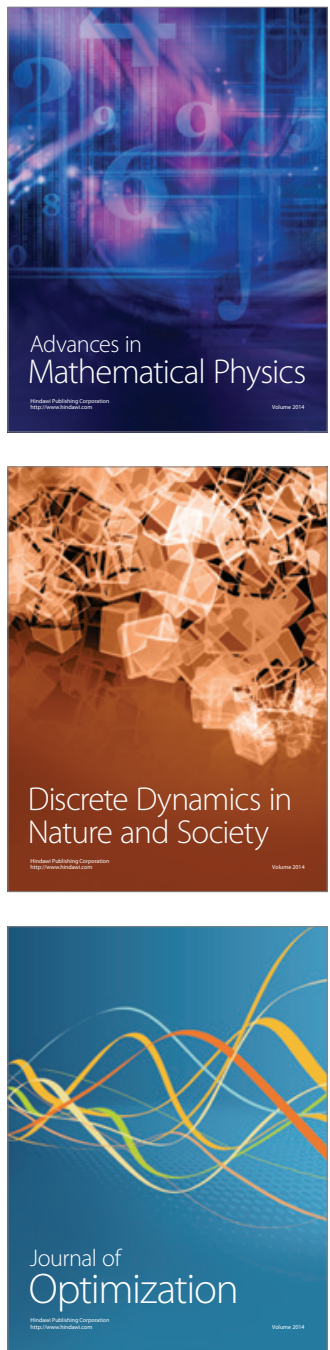